\title{
Protein Aggregation in the Cell Nucleus: Structure, Function and Topology
}

\author{
Anna von Mikecz* \\ Institut für umweltmedizinische Forschung (IUF) at Heinrich-Heine-University Düsseldorf, Auf'm Hennekamp 50, \\ 40225 Düsseldorf, Germany
}

\begin{abstract}
The nucleus represents a cellular control unit that regulates all events concerning the storage and processing of DNA and RNA. It is organized by highly crowded, dynamic assemblies of proteins and nucleic acids in molecular machines, ribonucleoprotein complexes, clusters of ongoing nuclear processes, nuclear bodies, and chromatin. This review discusses the occurrence of nuclear protein aggregation with special emphasis on the functional architecture of the nucleus, and quality control by the ubiquitin-proteasome system.
\end{abstract}

Keywords: Amyloid, gene expression, Huntington's disease, neurodegeneration, nucleus, polyglutamine, protein aggregation, trinucleotide repeat, ubiquitin-proteasome system.

\section{THE CELL NUCLEUS: DYNAMIC MACROMOLE- CULE CLUSTERING PROVIDES FUNCTION}

Nuclear function is embedded in microscopically discernible structural compartments. These extend from the nuclear envelope at the boundary between the nucleus and the cytoplasm, through internal nuclear bodies (NBs) such as the nucleolus, to the chromosomes within which histone proteins and DNA are packaged into nucleosomes and distinctly compacted chromatin fibers. The most prominent NBs include the nucleolus [1], which is the site of transcription of ribosomal RNA, the splicing factor compartments or speckles [2], which act as a storage site for spliceosomal components, Cajal NBs [3] and PML NBs [4], both of unknown function.

While genomes are defined by their primary sequence the key question that emerged after recent sequencing of organisms, including Homo sapiens, is how genome function is integrated into the architectural framework of the cell nucleus. The prevalent concept is that many of these subdomains are associated with specific genetic loci and that interactions between various domains are dynamic and can change in response to cellular signals [5-7]. A dynamic protein exchange occurs between nuclear domains and the surrounding nucleoplasm with exchange rates that depend on the functional activity of a nuclear protein and / or its participation in ribonucleoprotein complexes. Thus, the functional status of exchanging proteins critically determines the composition and morphology of subnuclear compartments [8]. A paradigm for nuclear structure function relationships is the nucleolus that forms by clustering of the RNA-polymerase Idependent transcription machinery at tandem repeats of ribosomal DNA in the so-called nucleolus organizer regions (NORs) during the start of interphase thereby initiating the

*Address correspondence to this author at the Institut fuer umweltmedizinische Forschung (IUF) at Heinrich-Heine-University Düsseldorf, Auf'm Hennekamp 50, 40225 Düsseldorf, Germany; Tel: ++49211-3389-358; E-mail: mikecz@tec-source.de;

Web: www.tec-source.de/mikecz synthesis of ribosomal RNA, and disaggregates at the start of mitosis [1]. Nucleolar decomposition is also observed as a direct consequence of inhibition of RNA polymerase I, e.g. ribosomal RNA transcription, by xenobiotics $[1,9]$.

The structural dynamics of subnuclear compartments such as the nucleolus and nuclear bodies seem to allow for functional flexibility, but this flexibility requires regulation. Hundreds of macromolecules including chromatin modulators, transcription factors, nuclear receptors and ribonucleoprotein particles (RNPs), cluster to form the molecular machineries for replication, DNA repair, transcription, RNA splicing, and ribosome biogenesis. How are these clusters disassembled and turned off in order to enable the sequential steps in gene expression to proceed? This question is further complicated by the fact that macromolecules in the nucleus are highly concentrated (approximately $100 \mathrm{mg} / \mathrm{ml}$ ) which is likely to cause strong crowding forces. While molecular crowding may represent the mechanism of nuclear compartment formation $[10,11]$, it may at the same time promote formation of aberrant and insoluble protein aggregates in the nucleoplasm [12]. Consistent with this idea it is important to note that fibrillation-prone proteins containing homopeptide repeat stretches such as polyglutamine (polyQ) are essential components of macromolecular complexes that sustain gene expression as well as aberrant nucleoplasmic protein aggregates (Fig. (1); [13-15]). The transcription initiation complex includes proteins with unexpanded polyQ repeats that function as transcriptional co-activators, transcription factors, nuclear receptors, and general transcription factors.

\section{THE UBIQUITIN-PROTEASOME-SYSTEM PARTICI- PATES IN NUCLEAR FUNCTION}

A growing body of evidence indicates that the ubiquitin proteasome system (UPS) is intimately involved in gene expression control at the transcription level and quality control in the cell nucleus [16-19]. In eukaryotic cells, a large ATPdependent proteolytic machine, the $26 \mathrm{~S}$ proteasome [20], 


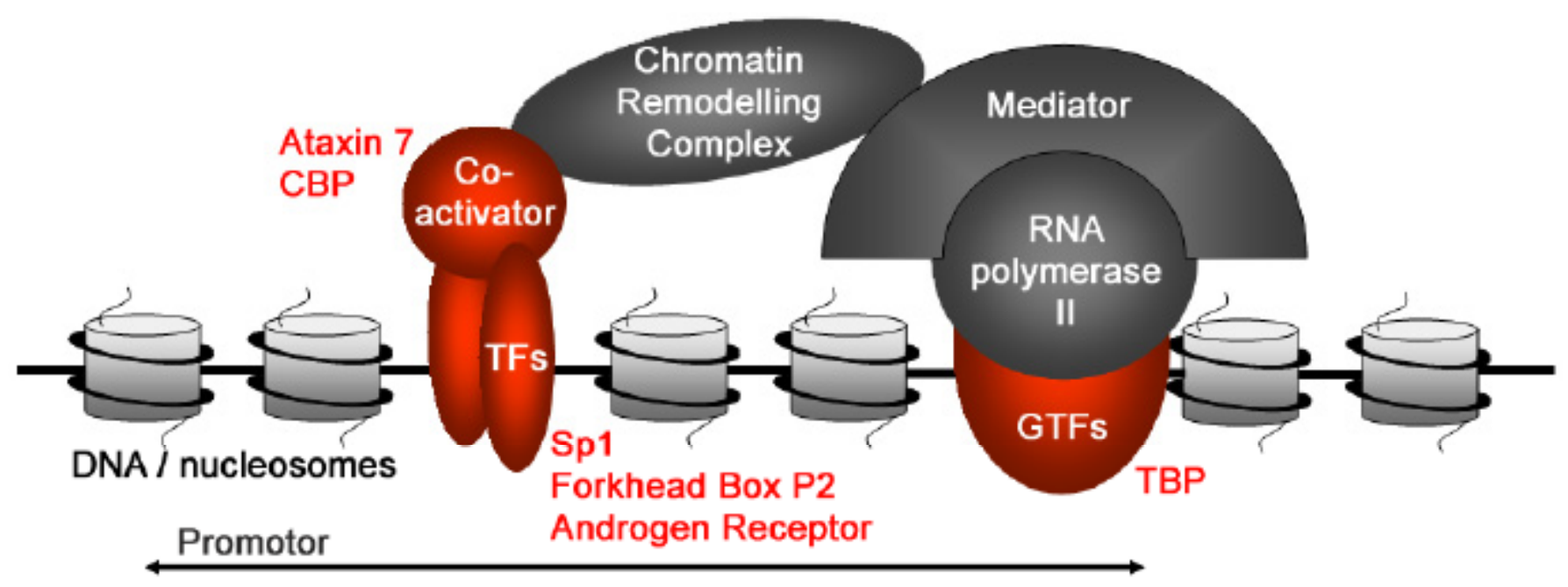

Fig. (1). The initiation complex of eukaryotic RNA-polymerase II-dependent transcription (schematic representation). Components include proteins with unexpanded, homopolymeric polyglutamine repeats such as (i) coactivators ataxin 7, and CBP, (ii) transcription factors Sp1, forkhead box P2, and androgen receptor, and (iii) general transcription factor TBP (red). CBP, CREB binding protein; GTFs, general transcription factors; TBP, TATA box binding protein; TFs, transcription factors.

prevents accumulation of non-functional, potentially toxic proteins. The $26 \mathrm{~S}$ proteasome is a self-compartmentalized multisubunit protease complex that degrades substrates which are post-translationally tagged by a polyubiquitin chain [20]. The $26 \mathrm{~S}$ proteasome is composed of a $20 \mathrm{~S}$ catalytic core and two flanking $19 \mathrm{~S}$ regulatory complexes constituting the structure 19S-20S-19S. According to the current conception polyubiquitinated proteins are (i) recognized by the $19 \mathrm{~S}$ regulatory subunits, (ii) unfolded, (iii) channeled via a central passageway into the degradation chamber, (iv) degraded into peptides, and (v) released through the entry channel [21]. The polyubiquitinated substrates seem to allosterically activate proteasomal degradation by binding to the 19S regulator, which in turn induces stabilization of gate opening and facilitation of substrate entry into the proteolytic chamber [22].

Dynamic nuclear processes have to be regulated and controlled in order to maintain the function of the nucleus throughout interphase. More than twenty years ago, Franke and colleagues observed that proteasomes localize to the nuclei of Xenopus laevis oocytes and HeLa cells $[23,24]$. Varshavsky and co-workers showed that ubiquitin-dependent pathways play a role in cell cycle control and suggested that protein degradation is instrumental in regulation of gene expression [25]. The following decades brought about confirmation that components of the UPS indeed reside in the cell nucleus and that nuclear proteins are substrates for proteasomal degradation. However, export of nuclear proteins to the cytoplasm was considered to be required for their degradation. Prominent examples include the tumor suppressor p53 and cyclin-dependent kinase (CDK) inhibitor p27 [26-28]. Thus, research predominantly concentrated on cytoplasmic protein breakdown showing that this generates peptides for antigen presentation [29] and removes the bulk of newly synthesized, defective ribosomal products (DRIPs, $[30,31]$.

Recent work suggests direct engagement of a nuclear UPS (nUPS) in DNA repair, replication, transcription, and nuclear quality-control [13,15,18,32-36]. Proteasomal activity is localized in subnuclear compartments such as speckles, PML NBs and distinct proteolytic foci in euchromatic regions [15,36,37; Scharf and von Mikecz, unpublished results]. Proteasomal proteolysis in loosely packed euchromatin is consistent with the idea of an interaction of a nUPS with nuclear processes. Indeed, ubiquitin and the proteasome system are intimately involved in the regulation of gene expression control at the transcription level. The yeast transcription factor Gcn 4 binds to a cognate promoter region, activates Gcn4-responsive genes and is subsequently marked for destruction by $\mathrm{SCF}^{\mathrm{Cdc} 4}$, the ubiquitin ligase for Gen4. These events suggest that proteolysis is required to remove "spent" activators in order to reset the promoter. Indeed, degradation of Gcn 4 and the transcriptional activators Gal4 and Ino2/4 by the UPS stimulates expression of their targets. Moreover, studies have shown mutations in $\mathrm{SCF}^{\mathrm{Cdc} 4}$ and mutations in ubiquitin that prevent proteolysis also impair the transcription of Gen4 targets [38], confirming this idea. While a physical association with active transcription sites has not been observed during initiation and elongation [39; Scharf and von Mikecz, unpublished], it could be shown that proteasomes recruit to sites of stalled transcription machineries due to UV-induced DNA damage [40]. In contrast to fluorescence recovery after photobleaching (FRAP) analyses with ectopically expressed proteasomal subunit LMP2 that suggested a high mobility of proteasomes in the nucleus [41], proteasomal proteolytic activity seems to be localized to confined nuclear loci that partially overlap with nuclear subcompartments [36,42], and are generally immobile (Scharf and von Mikecz, unpublished).

In summary, it is now well established that the UPS is not only present in the nucleus, but an active player in nuclear processes. Compartmentalized proteolysis allows cells to regulate degradation of specific proteins by control of their subcellular localization. A tuned balance of ubiquitination and proteasome-dependent protein degradation of nuclear poteins in distinct nucleoplasmic proteolysis factories may be instrumental in nuclear function and, when 
deregulated, lead to abnormal protein aggregation in the cell nucleus. Aberrant nuclear protein aggregation may in turn be involved in the development of protein aggregation diseases such as polyglutamine disorders and other neurodegenerative conditions.

\section{NUCLEAR PROTEIN AGGREGATES: OCCURENCE}

If a native protein is damaged it generally converts to a misfolded conformation. Such a misfolded protein may be subjected to (i) chaperone-mediated refolding back into the native conformation, (ii) ubiquitination and subsequent degradation by the proteasome, (iii) cleavage and nucleocytoplasmic transport, or (iv) protein aggregation [19]. Chaperoneaided refolding results in rescue of the functional protein, whereas proteasome-dependent degradation, cleavage, subcellular transport, and accumulation in protein aggregates deprive proteins from their genuine cellular function. Proteasomal proteolysis clearly serves to prevent aberrant accumulation of misfolded proteins. The role of protein aggregation is less clear. Experimental evidence has been collected for both, aggregate toxicity [43-45] and protein aggregates as protective segregation domains [46-49].

The toxic role of protein aggregates has been deduced from the fact that they are microscopically discernible in numerous neurodegenerative diseases. Cytoplasmic Lewy bodies were discovered in patients with Parkinson's disease as early as the beginning of the 20th century [16]. Likewise, extracellular plaques and intracellular tangles occur in Alzheimer's, whereas polyglutamine disorders are characterized by cytoplasmic and nuclear inclusions. Polyglutamine disorders represent a subset of trinucleotide repeat diseases in which proteins with unstable, expanded homopolymeric amino acid repeats accumulate in distinct subcellular domains [50]. A hallmark of the most widely known polyglutamine disorder Huntington's disease (HD) is the cytoplasmic and nucleoplasmic aggregation of the gene product huntingtin, a $348 \mathrm{kDa}$ protein with unknown function. Spinocerebellar ataxias (SCAs) are characterized by nuclear inclusions of ataxin proteins or TATA box binding protein (TBP) with expanded polyglutamine repeats. Finally, in spinal and bulbar muscular atrophy (SBMA) mutant androgen receptor is accumulated in the cytoplasm and nucleoplasm [49,51].

\section{NUCLEAR PROTEIN AGGREGATES: COMPOSI- TION}

The main components that have been identified in nuclear protein aggregates include (i) proteins with instable, elongated trinucleotide repeats, (ii) proteins with trinucleotide repeats of normal length, (iii) elements of the UPS, (iv) heat shock proteins, and (v) proteins that normally localize to subnuclear domains such as the nucleolus, speckles, Cajal NBs and PML NBs (for a comprehensive listing see supplemental material of [15]). System biology-based approaches demonstrate that the polyglutamine protein huntingtin interacts amongst others with general transcription factor TBP, histone acetylase CREB binding protein, transcription factor SP1, histone acetylase HDAC1, and the signature protein of PML NBs PML, thus building an elaborate interac- tome [52]. While it is not clear whether all these interactions are of functional significance, it is important to note that components of this protein network are also components of nuclear protein aggregates [15].

Consistent with the idea of dynamic nuclear structures it was shown by fluorescence imaging of living cells that proteins which participate in nuclear protein inclusions such as molecular chaperones are not irreversibly sequestered into aggregates, but rather transiently associated [53]. In analogy to other dynamic subdomains of the nucleus exchange of inclusion components might depend on the functional status of the proteins and / or the aggregate (see above). Thus, chaperones could represent active and therefore transient passengers through nuclear protein aggregates. Since polyglutamine-induced inclusions form by sequential steps of amyloid-species such as small soluble aggregates, protofibrils, and mature fibrils [54,55] the dynamics of protein components may likewise depend on the progression of the aggregate structure. Small, amorphous aggregates may still provide the physicochemical conditions for component exchange, whereas mature amyloid fibrils or their accumulation in plaques may be characterized by irreversible protein sequestration.

Formation of amyloid-like intranuclear inclusions is experimentally inducible by addition of silica-nanoparticles (NPs) to cells in culture $[13,15]$. These nuclear protein aggregates exactly mimic their polyglutamine-induced counterparts with respect to biochemical properties and protein composition. Silica-NP-induced nuclear inclusions (i) stain with Congo red and thioflavin T, (ii) are insoluble as analysed by filter binding assays and density gradient centrifugation, (iii) and can be reduced by Congo red and trehalose [15; Chen and von Mikecz, unpublished]. As an advantage versus other experimental systems protein aggregation by nanoparticles allows for the characterization of endogenous aggregate components with a functional UPS in place. Thus, the silica-NP-induced inclusions contain polyglutamine proteins huntingtin, ataxin-3, TBP, and CREB binding protein, components of the UPS, and nuclear proteins such as fibrillarin, topoisomerase I, p80 coilin, and PML that are normally localized in nucleoli, Cajal NBs and PML NBs, respectively. Detailed localization by confocal microscopy shows that polyglutamine proteins occur in the center, whereas other nuclear proteins and proteasomes accumulate at the periphery of silica-NP-induced nuclear inclusions (Fig. 2). A similar aggregate morphology was reported in vitro and in vivo showing aggregated polyglutamine proteins engulfed in a coat of PML protein and close to Cajal NBs [56,57]. Localization of proteasomal activity revealed that one third of nanoparticle-induced protein aggregates are proteolytically active and that $90 \%$ of this subpopulation colocalized with PML [15].

\section{NUCLEAR PROTEIN AGGREGATES: PROTEO- LYTIC CENTERS}

A role for PML NBs in removal of excess and or misfolded proteins has been initially proposed for viral proteins. Yewdell and coworkers showed accumulation of mutated forms of influenza virus in PML NBs after inhibition of proteasome-dependent protein degradation [58]. 
A. aggregation of topoisomerase I with ...

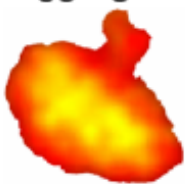

TBP

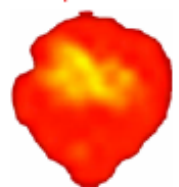

huntingtin

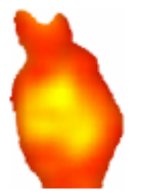

CBP

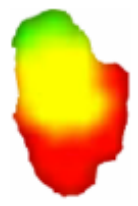

$\mathrm{PML}$

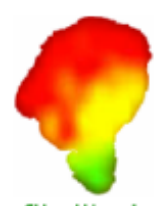

fibrillarin

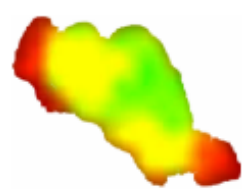

SC35

B.

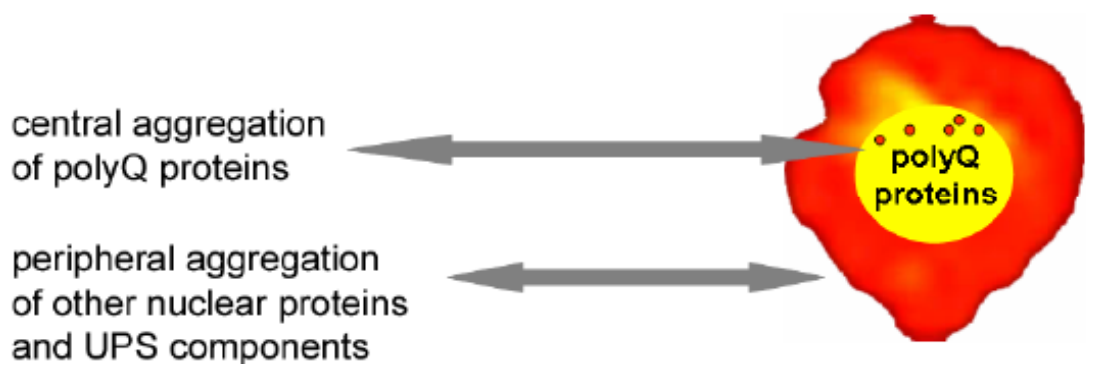

Fig. (2). (A) Confocal immunofluorescence of nuclear protein aggregates. Blow ups of double labelled topoisomerase I (red) and polyglutamine proteins or signature proteins of subnuclear domains (green). Merged areas of colocalization appear yellow. (B) Proposed mechanism of nuclear protein aggregation: Proteins with homopolymeric polyQ repeats form an aggregate-core, consequently recruiting components of ribonucleoprotein complexes and the UPS. CBP, CREB binding protein; polyQ, polyglutamine; SC35, splicing factor; TBP, TATA box binding protein; UPS, ubiquitin-proteasome system.

Due to viral nucleoprotein-induced recruitment of proteasomes and molecular chaperones to PML NBs the authors suggested that these NBs serve as proteolytic centers in the nucleus. But which features would make PML NBs suited as sites of protein degradation?

PML NBs are amorphous, spherical structures that can be localized by a variety of microscopical methods. To date approximately 78 protein components have been assigned to PML NBs [59; nuclear protein database: http://npd.hgu. mrc.ac.uk/index.html] with PML and Sp100 representing constitutive signature proteins, and Daxx, small ubiquitinlike modifier SUMO, Bloom syndrome gene product BLM and NDP55 present at endogenous expression levels. In contrast to nucleoli and Cajal NBs, PML NBs do not contain DNA or RNA, and are consistently devoid of ribonucleoprotein complexes. PML NBs are dynamic subnuclear domains that alter their number, size and components in response to diverse environmental stimuli such as viral infections, extracellular signalling, and genotoxic stress. However, under normal growth conditions a typical mammalian nucleus contains between 10 to 30 PML NBs sized between 0.3 and $1.0 \mu \mathrm{m}$ [60]. The residence times of PML, Sp100 and other components in their respective PML NB may last from several seconds to 1 hour [61] suggesting a constant exchange of constituents between the NB and the surrounding nucleoplasm. In contrast to its components the PML NB itself appears to be mostly static due to extensive contact to chromatin. A small and mobile subpopulation of PML NBs has been identified and it was suggested to function as a sensor that detects foreign and misfolded proteins, including viral proteins and protein aggregates [62].

The dynamic properties of their constituents and a subpopulation of the NBs together with results indicating colocalization of PML NBs with the UPS lead to the following hypothesis: PML NBs might detect foreign and / or misfolded proteins and promote their degradation by proteasomes. Consistent with this idea it was shown that the degradation of PML is correlated to colocalization between PML NBs and proteasomes [37], degradation of a fluorescent model substrate can be partially localized at PML NBs [36], and the majority of silica-NP-induced protein aggregates which are proteolytically active are coated by PML in a ring-shaped pattern [15]. The model of PML NBs as proteolytic centers is made even more appealing by the observation that overexpressed proteins regularly form nuclear protein aggregates and colocalize with PML $[4,63]$.

An interaction between PML NBs and protein aggregation was observed in vivo and in vitro. In the brains of patients with spinocerebellar ataxia 7 , and respective mouse models mutated ataxin 7 (with an expanded polyglutamine tract) colocalized with PML NBs $[64,65]$. Consistent with a protective, proteolytic role it was shown that PML NBs are associated with early steps of polyglutamine-associated amyloid formation [64]. In cell culture models expression of mutant ataxin-1 and -3 induced aberrant distribution of PML in the nucleus [66]. In reverse, overexpression of PML isoform IV promoted the formation of NBs enriched in UPS components [65]. Such NBs recruited ataxin-7, but prevented formation of insoluble protein aggregates by proteasomedependent proteolysis. Together with results that detect PML in proteolytically active nuclear inclusions [15] a picture emerges where PML NBs may serve as proteolytic centers for misplaced and / or misfolded proteins in the cell nucleus, thus preventing further steps of protein aggregation which would eventually give rise to insoluble, irreversible inclusions. The dynamic nature of PML NBs with respect to their protein components, and the lack of ribonucleoprotein complexes may allow for a promiscuous and continuous exchange of potential proteasome substrates. Thus, PML NBs may at the same time be proteolytic centers and nuclear protein aggregates depending on the definition and foremost on a fine tuned regulation by the UPS, e.g. proteasomedependent proteolytic activity. 


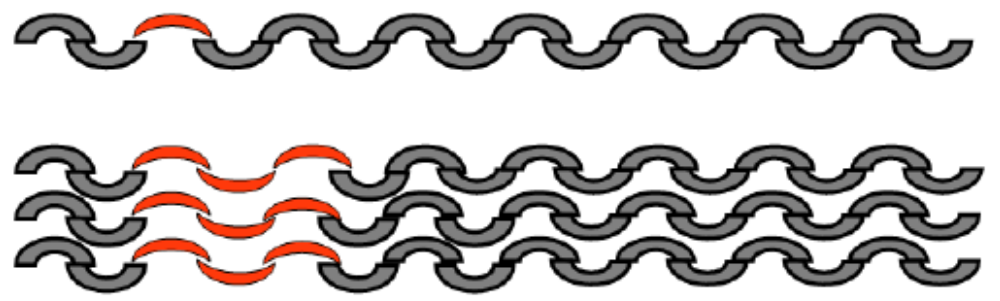

\author{
protein with UNexpanded \\ polyQ repeat
}

proteins with expanded

polyQ repeats aggregate

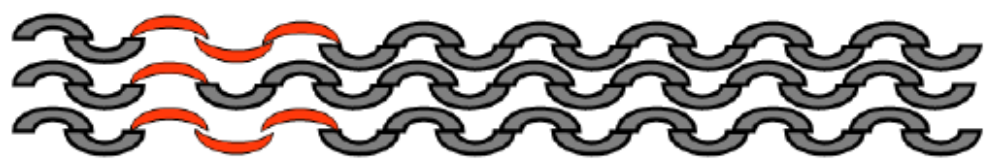

proteins with expanded and UNexpanded polyQ repeats aggregate

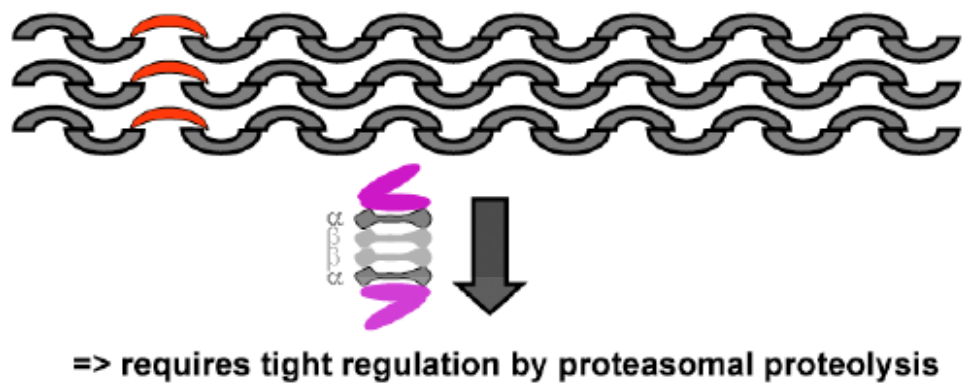

all proteins with polyQ repeats aggregate

Fig. (3). Intranuclear fibrillation of proteins with unexpanded or expanded homopolymeric polyglutamine (polyQ, red) repeats and combinations thereof is under constitutive control by proteasome-dependent proteolysis (hypothesis).

\section{PERSPECTIVES}

Protein aggregation in the cell nucleus seems to occur spontaneously in brains of patients with neurodegenerative triplet repeat diseases and respective animal models. In cell culture nuclear protein inclusions can be induced by environmental stressors, including silica-NPs. From cell-based models we may learn more about the molecular mechanisms and the role of protein aggregation. Recent results indicate that nucleoplasmic protein aggregates are not simply "junkyards" for excess and / or misfolded protein species. The UPS acts by means of proteasome-dependent protein degradation in one third of these "junkyards". Due to the dynamic and crowded topology of the nucleus that is required for processes such as transcription, functional multiprotein complexes (Fig. 1) may always be prone to aggregation, and without regulation by proteasomal proteolysis in danger to undergo the serial steps of polyQ fibrillation (Fig. 3). Thus, a functional nuclear ribonucleoprotein cluster may resemble a protein aggregate in protein composition, but differ in its proteasomal activity. Likewise, the presence of molecular chaperones may decide the fate of nuclear protein aggregates. Towards a better understanding of nuclear protein aggregation and its anticipated biological function we need more systematic approaches to identify aggregate component networks in correlation to both, detailed analysis of the biophysical protein fibrillation (or amyloid) status and activity of the UPS (Fig. 3). Development of relevant nuclear substrates for proteasomal degradation will be instrumental to pinpoint proteolytic centers, including protein aggregates, and determine their influence on neurons as well as neurodegeneration. An equally important goal in order to elucidate the pathogenesis of neurodegenerative polyglutamine diseases is a better understanding of amyloidogenesis in the environment of the nucleus.

\section{ACKNOWLEDGEMENTS}

AvM is supported by the German Science Foundation (DFG) through grants SFB 728 and GRK 1033, the German Federal Ministry for the Environment and Reactor Safety (BMU), and the German Foundation for Systemic Sclerosis (DSS).

\section{REFERENCES}

[1] Sirri V, Urcuqui-Inchima S, Roussel P, Hernandez-Verdun D. Nucleolus: the fascinating nuclear body. Histochem Cell Biol 2008; 129: 13-31.

[2] Spector DL. Nuclear organization of pre-mRNA processing. Curr Opin Cell Biol 1993; 5: 442-7.

[3] Cioce M, Lamond A. Cajal bodies: a long history of discovery. Annu Rev Cell Dev Biol 2005; 21: 105-31.

[4] Bernardi R, Pandolfi PP. Structure, dynamics and functions of promyelocytic leukaemia nuclear bodies. Nat Rev Mol Cell Biol 2007; 8: 1006-16.

[5] Schul W, de Jong L, van Driel R. Nuclear neighbours: the spatial and functional organization of genes and nuclear domains. J Cell Biochem 1998; 70: 159-71.

[6] Misteli T. Protein dynamics: implications for nuclear architecture and gene expression. Science 2001; 291: 843-7.

[7] Wachsmuth M, Caudron-Herger M, Rippe K. Genome organization: balancing stability and plasticity. Biochim Biophys Acta 2008; 1783: 2061-79.

[8] Misteli T. Beyond the sequence: cellular organization of genome function. Cell 2007; 128: 787-800. 
[9] Chen M, von Mikecz A. Specific inhibition of rRNA transcription and dynamic relocation of fibrillarin induced by mercury. Exp Cell Res 2000; 259: 225-38.

[10] Hancock R. A role for macromolecular crowding effects in the assembly and function of compartments of the nucleus. J Struct Biol 2004; 146: 281-90.

[11] Marenduzzo D, Finan K, Cook P. The depletion attraction: an underappreciated force driving cellular organization. J Cell Biol 2006; 175: 681-6.

[12] Ellis RJ, Minton AP. Protein aggregation in crowded environments. Biol Chem 2006; 387: 485-97.

[13] Chen M, von Mikecz A. Formation of nucleoplasmic protein aggregates impairs nuclear function in response to $\mathrm{SiO}_{2-}$ nanoparticles. Exp Cell Res 2005; 305: 51-62.

[14] Faux NG, Bottomley SP, Lesk AM, et al. Functional insights from the distribution and role of homopeptide repeat-containing proteins. Genome Res 2005; 15: 537-51.

[15] Chen M, Singer L, Scharf A, von Mikecz A. Nuclear polyglutamine-containing protein aggregates as active proteolytic centers. J Cell Biol 2008; 180: 697-704.

[16] Mayer RJ. The meteoric rise of regulated intracellular proteolysis. Nat Rev Mol Cell Biol 2000; 1: 145-8.

[17] Goldberg AL. Protein degradation and protection against misfolded or damaged proteins. Nature $2003 ; 426: 895-9$.

[18] von Mikecz A. The nuclear ubiquitin-proteasome system (nUPS). J Cell Sci 2006; 119: 1977-84.

[19] Schwartz AL, Ciechanover A. Targeting proteins for destruction by the ubiquitin system: implications for human pathobiology. Annu Rev Pharmacol Toxicol 2009; 49:73-96.

[20] Voges D, Zwickl P, Baumeister W. The 26S proteasome: a molecular machine designed for controlled proteolysis. Annu Rev Biochem 1999; 68: 1015-68.

[21] Finley D. Ubiquitin chained and crosslinked. Nat Cell Biol 2002; 4: E121-E123.

[22] Bech-Otschir D, Helfrich A, Enenkel C, et al. Polyubiquitin substrates allosterically activate their own degradation by the $26 \mathrm{~S}$ proteasome. Nat Struct Mol Biol 2009; 16: 219-25.

[23] Hugle B, Kleinschmidt JA, Franke WW. The $22 \mathrm{~S}$ cylinder particles of Xenopus laevis. II. Immunological characterization and localization of their proteins in tissues and cultured cells. Eur J Cell Biol 1983; 32: 157-63.

[24] Kleinschmidt JA, Hugle B, Grund C, Franke WW. The 22S cylinder particles of Xenopus laevis. I. Biochemical and electron microscopic characterization. Eur J Cell Biol 1983; 32: 143-56.

[25] Finley D, Ciechanover A, Varshavsky A. Thermolability of ubiquitin-activating enzyme from the mammalian cell cycle mutant ts85. Cell 1984; 37: 43-55.

[26] Freedman DA, Levine AJ. Nuclear export is required for degradation of endogenous $\mathrm{p} 53$ by $\mathrm{Mdm} 2$ and human papillomavirus E6. Mol Biol Cell 1998; 18: 7288-7293.

[27] Kamura $\mathrm{T}$, Hara $\mathrm{T}$, Matsumoto $\mathrm{M}$, et al. Cytoplasmic ubiquitin ligase KPC regulates proteolysis of p27(Kip1) at G1 phase. Nat Cell Biol 2004; 6: 1229-35.

[28] Pines J, Lindon C. Proteolysis: anytime, any place, anywhere? Nat Cell Biol 2005; 7: 731-5.

[29] Rock KL, Gramm C, Rothstein L, et al. Inhibitors of the proteasome block the degradation of most cell proteins and the generation of peptides presented on MHC class I molecules. Cell 1994; 78: 761-71.

[30] Reits EA, Vos JC, Gromme M, Neefjes J. The major substrates for TAP in vivo are derived from newly synthesized proteins. Nature 2000; 404: 774-8.

[31] Schubert U, Anton LC, Gibbs J, Norbury CC, Yewdell JW, Bennink JR. Rapid degradation of a large fraction of newly synthesized proteins by proteasomes. Nature 2000; 404: 770-4.

[32] Mendez J, Zou-Yang XH, Kim S-Y, Hidaka M, Tansey WP, Stillman B. Human origin recognition complex large subunit is degraded by ubiquitin-mediated proteolysis after initiation of DNA replication. Mol Cell 2002; 9: 481-91.

[33] Baker SP, Grant PA. The proteasome: not just degrading anymore. Cell 2005; 123: 361-3.

[34] Gardner RG, Nelson ZW, Gottschling DE. Degradation-mediated protein quality control in the nucleus. Cell 2005; 120: 803-15.

[35] Pfander B, Moldovan G-L, Sacher M, Hoege C, Jentsch S. Sumomodified PCNA recruits Srs2 to prevent recombination during $\mathrm{S}$ phase. Nature 2005; 436: 428-33.
[36] Rockel TD, Stuhlmann D, von Mikecz A. Proteasomes degrade proteins in focal subdomains of the human cell nucleus. J Cell Sci 2005; 118: 5231-42.

[37] Rockel TD, von Mikecz A. Proteasome-dependent processing of nuclear proteins is correlated with their subnuclear localization. J Struct Biol 2002; 140: 189-99.

[38] Lipford JR, Smith GT, Chi Y, Deshaies R. A putative stimulatory role for activator turnover in gene expression. Nature 2005; 438: 113-6.

[39] Gonzalez F, Delahodde A, Kodadek T, Johnston SA. Recruitment of a $19 \mathrm{~S}$ proteasome subcomplex to an activated promoter. Science 2002; 296: 548-50.

[40] Gillette TG, Gonzalez F, Delahodde A, Johnston SA, Kodadek T. Physical and functional association of RNA polymerase II and the proteasome. Proc Natl Acad Sci USA 2004; 101: 5904-9.

[41] Reits EA, Benham AM, Plougastel B, Neefjes J, Trowsdale J. Dynamics of proteasome distribution in living cells. EMBO J 1997; 16: 6087-94.

[42] Scharf A, Rockel T, von Mikecz A. Localization of proteasomes and proteasomal proteolysis in the mammalian cell nucleus by systematic application of immunocytochemistry. Histochem Cell Biol 2007; 127: 591-601.

[43] Davies SW, Turmaine M, Cozens BA, et al. Formation of neuronal intranuclear inclusions underlies the neurological dysfunction in mice transgenic for the HD mutation. Cell 1997; 90: 537-48.

[44] Yang W, Dunlap J R, Andrews R B, Wetzel R. Aggregated polyglutamine peptides delivered to nuclei are toxic to mammalian cells. Hum Mol Genet 2002; 11: 2905-17.

[45] Kayed R, Head E, Thompson JL, et al. Common structure of soluble amyloid oligomers implies common mechanism of pathogenesis. Science 2003; 300: 486-9.

[46] Gutekunst CA, Li SH, Yi H, et al. Nuclear and neuropil aggregates in Huntington's disease: relationship to neuropathology. J Neurosci 1999; 19: 2522-34.

[47] Arrasate M, Mitra S, Schweitzer ES, Segal MR, Finkbeiner S. Inclusion body formation reduces levels of mutant huntingtin and the risk of neuronal death. Nature 2004; 431: 805-10.

[48] Bodner RA, Outeiro TF, Altmann S, et al. Pharmacological promotion of inclusion formation: a therapeutic approach for Huntington's and Parkinson's diseases. Proc Natl Acad Sci USA 2006; 103: 4246-51.

[49] Slow EJ, Graham RK, Hayden MR. To be or not to be toxic: aggregations in Huntington and Alzheimer disease. Trends Genet 2006; 22: 408-11.

[50] Orr HT, Zoghbi HY. Trinucleotide repeat disorders. Annu Rev Neurosci 2007; 30: 575-621

[51] Adachi H, Katsuno M, Minamiyama M, et al. Widespread nuclear and cytoplasmic accumulation of mutant androgen receptor in SBMA patients. Brain 2005; 128: 659-70.

[52] Goehler $\mathrm{H}$, Lalowski M, Stelzl U, et al. A protein interaction network links GIT1, an enhancer of huntingtin aggregation, to Huntington's disease. Mol Cell 2004; 15: 853-65.

[53] Kim S, Nollen EAA, Kitagawa K, Bindokas VP, Morimoto RI. Polyglutamine protein aggregates are dynamic. Nat Cell Biol 2002; 4: 826-31.

[54] Dobson CM. Protein foldung and misfolding. Nature 2003; 426 : 884-90.

[55] Chiti F, Dobson CM. Protein misfolding, functional amyloid, and human disease. Annu Rev Biochem 2006; 75: 333-66.

[56] Yamada $\mathrm{M}$, Sato $\mathrm{T}$, Shimohata $\mathrm{T}$, et al. Interaction between neuronal intranuclear inclusions and promyelocytic leukemia protein nuclear and coiled bodies in CAG repeat diseases. Am J Pathol 2001; 159:1785-95.

[57] Hazeki N, Tsukamoto T, Yazawa I, et al. Ultrastructure of nuclear aggregates formed by expressing an expanded polyglutamine. Biochem Biophys Res Commun 2002; 294:429-40.

[58] Anton LC, Schubert U, Bacík I, et al. Intracellular localization of proteasomal degradation of a viral antigen. J Cell Biol 1999; 146: $113-24$.

[59] Hofmann T, Will H. Body language: the function of PML nuclear bodies in apoptosis regulation. Cell Death Differ 2003; 10: 12901299.

[60] Spector DL. Nuclear domains. J Cell Sci 2001; 114: 2891-3.

[61] Weidtkamp-Peters S, Lenser T, Negorev D, et al. Dynamics of components exchange at PML nuclear bodies. J Cell Sci 2008; 121: $2731-43$. 
[62] Muratani M, Gerlich D, Janicki SM, Gebhard M, Eils R, Spector DL. Metabolic-energy-dependent movement of PML bodies within the mammalian cell nucleus. Nat Cell Biol 2002; 4: 106-10.

[63] Fu L, Gao Y-S, Sztul E. Transcriptional repression and cell death induced by nuclear aggregates of non-polyglutamine protein. Neurobiol Dis 2005; 20: 656-65.

[64] Takahashi J, Fujigasaki H, Iwabuchi K, et al. PML nuclear bodies and neuronal intranuclear inclusion in polyglutamine diseases. Neurobiol Dis 2003; 13: 230-7.
[65] Janer A, Martin E, Muriel MP, et al. PML clastosomes prevent nuclear accumulation of mutant ataxin-7 and other polyglutamine proteins. J Cell Biol 2006; 174: 65-76

[66] Skinner PJ, Koshy BT, Cummings CJ, et al. Ataxin-1 with an expanded glutamine tract alters nuclear matrix-associated structures. Nature 1997; 389: 971-4.

(C) Anna von Mikecz; Licensee Bentham Open.

This is an open access article licensed under the terms of the Creative Commons Attribution Non-Commercial License (http://creativecommons.org/licenses/by$\mathrm{nc} / 3.0 /$ ), which permits unrestricted, non-commercial use, distribution and reproduction in any medium, provided the work is properly cited. 